\title{
VOLUME FLOW RATE MEASUREMENT IN VERTICAL OIL-IN-WATER PIPE FLOW USING ELECTRICAL IMPEDANCE TOMOGRAPHY AND A LOCAL PROBE
}

\author{
$\mathrm{Hua} \mathrm{Li}^{a}$, Mi Wang ${ }^{a}$, Ying-Xiang $\mathrm{Wu}^{b}$ and Gary Lucas ${ }^{c}$ \\ ${ }^{a}$ Institute of Particle Science and Engineering, University of Leeds, Leeds LS2 9JT, UK. \\ Email:prehli@leeds.ac.uk,m.wang@leeds.ac.uk \\ ${ }^{b}$ Institute of Mechanics, Chinese Academy of Sciences, Beijing 100080, China. Email: \\ yxwu@imech.ac.cn \\ ${ }^{c}$ School of Computing and Engineering, University of Huddersfield, Huddersfield HDI \\ 3DH, UK. Email: g.lucas@hud.ac.uk
}

\begin{abstract}
This paper presents the use of a high performance dual-plane electrical impedance tomography (EIT) system and a local dual-sensor conductance probe to measure the vertical upward co-current oil-in-water pipe flows.

Experiments were carried on a flow loop with a transparent $2.5 \mathrm{~m}$ long, $80 \mathrm{~mm}$ inner diameter test section using kerosene and tap water. The flow conditions were predominantly of the dispersed type with the non-slip oil volume fraction of $9.1 \%, 16.7 \%$ and $23.1 \%$ respectively, and with two groups of different mixture velocities. A sensitivity coefficient back-projection (SBP) algorithm was adopted to reconstruct the flow distributions from the EIT measurement data, and then the oil in-situ volume fraction was calculated based on Maxwell relationship with temperature compensation. The oil velocity distribution was obtained using a pixel to pixel cross correlation method. A local intrusive conductance probe was adopted to supply a reference measurement of oil volume fraction and velocity profiles.

The oil volume fraction and velocity distributions from the two techniques were compared, and good agreement was found. A further calculation of the water velocity distributions and flowrates was implemented through the drift flux approach, and the results were analysed and discussed.
\end{abstract}

KEYWORDS: Volume fraction and velocity distributions, Oil-in-water flow, Electrical impedance tomography, Local probe

\section{INTRODUCTION}

Two phase flow of oil and water is commonly observed in the petroleum industry, where they are produced and transported together. For example, oil water flow in wellbore is often encountered when oil fields mature and the coproduction of water generally increases with time. However, such flows have not been studied to the same extent of gas-liquid flows.

In petroleum engineering applications, the flow pattern, the holdup and velocity of the two phases are very important hydrodynamic features. The complexity of two phase flows lies in the various configurations of interface distribution and non-uniform velocity profiles, and it constitutes a still unresolved issue for the petroleum industry. 
Process Tomography is an emerging measurement technology with applications in a broad range of industries. It's non-intrusive and provides a means of obtaining detailed local flow information such as phase and velocity distributions, which can help us to understand flow processes better and is beneficial in process monitoring and control. Due to its capability of obtaining local detailed flow distribution, it's promising to overcome the main challenge of multiphase flow measurement. However, only a few investigations could be found in oilwater flow, and even less with the electrical tomography.

In two-phase flow metering, it's usually hard to obtain all parameters of both phases. Thus, modelling techniques are also required to predict and interpret the results. In this study, a drift-flux approach was adopted to anzlyse the velocity slippage phenomenon. The drift-flux model proposed by Zuber and Findlay (1965) is widely used to represent two and three-phase flow in pipes and wellbores. It has been refined by Flores et al. (1998), Hasan and Kabir (1999) for modelling oil-water flow in vertical and deviated wellbores, and their investigation also showed significant slippage happed in water-dominated flow patterns.

The objective of this work is to investigate and validate the application of EIT system for metering such flows as oil-in-water vertical pipe flow, in which the continuous component is electrical conductive. The local volume fraction distributions obtained using EIT can be a certain level of erroneous, because they are highly sensitive to such factors as the accuracy of the electrical measurements made at the system boundary and the image reconstruction algorithm that is used (Dickin et al., 1993, p. 1883). At the presence of measurement error of $1 \%$, the conductivity error could up to $10 \%$ subjecting to the magnitude of conductivity charge (Wang et al., 1999, p. 49). Given the fact as above, it's important to have reference measurements with an independent technique. A local intrusive dual-sensor conductance probe was employed for this purpose in this study.

\section{EXPERIMENTAL SET-UP AND DATA PROCESSING}

\subsection{The flow loop}

The experiments were carried in a flow loop built at University of Huddersfield with a $2.5 \mathrm{~m}$ long, $80 \mathrm{~mm}$ internal diameter, transparent, vertical working section, which is shown in Fig. 1. Water and oil were pumped into the base of the working section via different branches with turbine meters respectively, which enabled the water volumetric flow rate $Q_{\mathrm{w}}$ and the oil volumetric flow rate $Q_{\mathrm{o}}$ to be measured.

Two groups of tests were carried out with water flow rates at 5 and $7.5 \mathrm{~m}^{3} / \mathrm{h}$, and oil flow rates at $0.5 / 1 / 1.5 \mathrm{~m}^{3} / \mathrm{h}$ and $0.75 / 1.5 / 2.25 \mathrm{~m}^{3} / \mathrm{h}$ respectively, which realised the flow conditions predominantly of the dispersed type with the non-slip oil volume fraction of $9.1 \%, 16.7 \%$ and $23.1 \%$ respectively, and with two groups of different mixture velocities.

\subsection{The EIT system and data processing}

A new EIT system for online measurement of two-phase flows has been developed at Leeds University, which is capable of metering axial velocity of $10 \mathrm{~m} / \mathrm{s}$ with $5 \%$ velocity measurement resolution (Wang et al., 2005, p. 289).

The distance between the dual planes of the EIT sensor with 16 electrodes per sensing plane was $30 \mathrm{~mm}$. A total of 8190 dual-frames of voltage measurements were acquired for each 
flow condition, which took $8.96 \mathrm{~s}$ at a speed of 914.3 dual-frames per sec (dfps). A sensitivity coefficient back-projection (SBP) algorithm was adopted to reconstruct flow images for its high speed, which adopted 316 square meshes.

\subsubsection{Concentration calculation}

With conductivity distributions from EIT, the oil concentration distributions (expressed as oil volume fraction, $\alpha_{0}$ ) can be determined by applying the Maxwell relationship (Maxwell 1881):

$$
\alpha_{o}=\frac{2 \sigma_{w}-2 \sigma_{m}}{2 \sigma_{w}+\sigma_{m}}
$$

where $\sigma_{\mathrm{w}}$ is the conductivity of water, and $\sigma_{\mathrm{m}}$ is the local mixture conductivity, and this is a simplified form in consideration of the conductivity of oil being zero.

The reconstruction algorithm uses the voltage relative changes between a measurement profile acquired from a flow with concentration change and a reference profile acquired from a flow with water only. Since the water temperature, therefore the conductivity, usually arises during the experimental process, and reference profile has to be compensated based on the water temperature. The relationship between voltage and temperature can be approximated as (Wang et al., 2003, p. 854):

$$
V_{T_{2}}=V_{T_{1}} e^{-0.0243\left(T_{2}-T_{1}\right)}
$$

where $V_{\mathrm{T} 1}$ and $V_{\mathrm{T} 2}$ are the voltages responded from a linear system at temperatures $T_{1}$ and $T_{2}$, respectively.

\subsubsection{Velocity calculation}

The axial flow velocity distributions can be estimated by a direct cross-correlation method, as given in eq. (3):

$$
R_{12}^{(k)}(n)=\sum_{m=1}^{k} f_{1}(m) f_{2}(m+n)
$$

where $k$ is the sample length, $n$ is the offset number and $f_{1}(m) \& f_{2}(m)$ are the $m^{\text {th }}$ up-flow and down-flow images respectively. Eq. (3) can be simply implemented online by updating the $R_{12}^{\mathrm{k}}(n)$ with the new $(k+1)^{\text {th }}$ images, as described in the following:

$$
R_{12}^{(k+1)}(n)=R_{12}^{(k)}(n)+f_{1}(k+1) f_{2}(k+1+n)
$$

This implementation can save calculation time and reduce memory size greatly (Wang et al., 2005, p. 289).

\subsubsection{Volumetric flow rate calculation}

The phase volumetric flow rate $Q_{\mathrm{i}}$ can be obtained by integrating the product of the local volume faction $\alpha_{i}$ and axial velocity $v_{i}$ of the specified phase in the flow cross section according to the equation: 


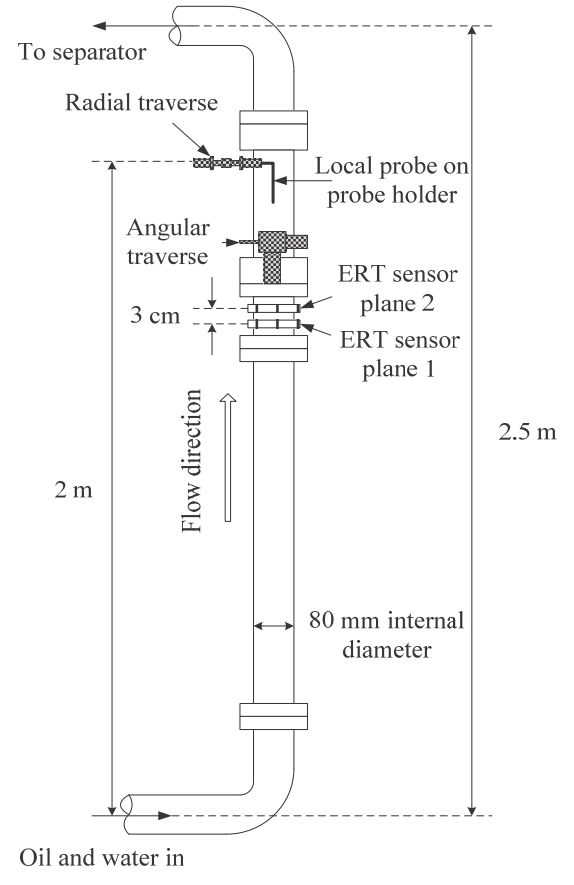

Figure 1. Schematic of the test section

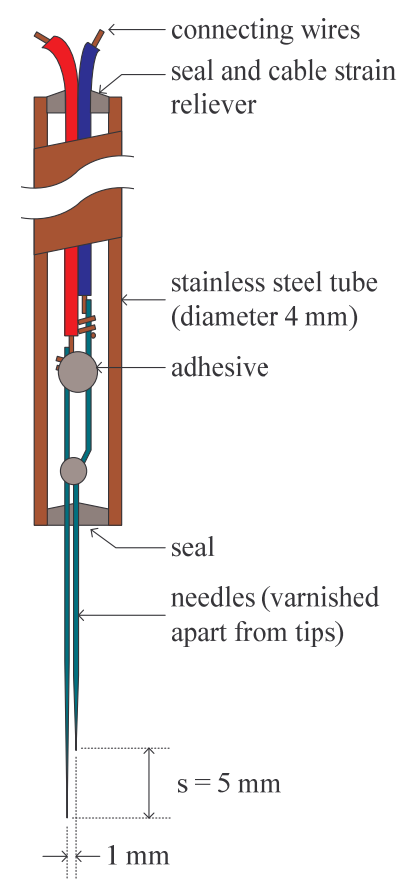

Figure 2. Construction of the probe

$$
Q_{i}=\int_{A} \alpha_{i} v_{i} \mathrm{~d} A
$$

where $A$ denotes the cross section area.

\subsection{The local dual-sensor conductance probe}

The dual sensor probe was constructed at University of Huddersfield. Each probe was manufactured from two stainless steel acupuncture needles, which were $0.3 \mathrm{~mm}$ in diameter and were mounted inside a stainless steel tube with an outer diameter of $4 \mathrm{~mm}$ as shown in Fig. 2 (Lucas et al., 2004, p. 271). Each acupuncture needle was coated with waterproof paint and insulating varnish except the very tip of the needle. The two needle tips of the probe are separated by an axial distance $s$ of $5 \mathrm{~mm}$ and a lateral distance of $1 \mathrm{~mm}$.

\subsubsection{The traversing mechanism}

The dual sensor conductance probe was mounted in a fully automated, two-axis traversing mechanism which enabled the probe to be moved to different spatial location in the measurement plane. In the experiments, the probe was traversed following two sets of parameters. One is across 4 equispaced diameters with 15 equispaced locations on each diameter giving a total of 57 distinct measurement locations respectively, the other is 8 equispaced diameters with 11 equispaced locations on each diameter giving a total of 81 distinct measurement locations. At each measurement location, and for each flow condition, data were acquired from the dual sensor probe for a period of $30 \mathrm{~s}$. 


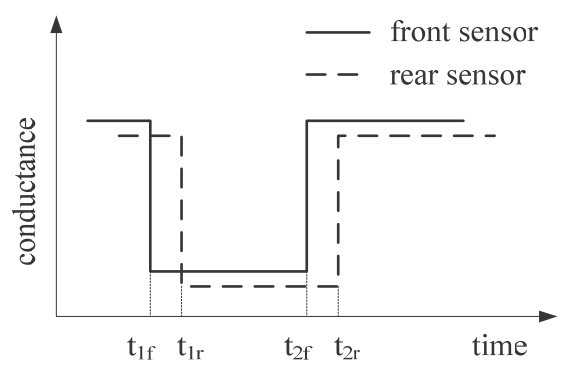

Figure 3. Ideal signals when an oil bubble striking the probe

\subsubsection{The operational principle}

The principle of operation can be briefly explained as follows (Lucas et al., 2004, p. 271). Consider an idealized situation in a vertical upward oil-water bubble flow in which the bubbles are assumed to travel in the axial direction only. When the surface of an oil bubble makes first contact with the upstream (front) sensor at time $t_{1 \mathrm{f}}$, the measured conductance at the front sensor will fall sharply (see Fig. 3) as it is immersed in oil instead of water. Then when the front sensor makes last contact with the surface of the bubble at time $t_{2 \mathrm{f}}$ (i.e., the time at which the bubble leaves the front sensor), the measured conductance at the front sensor will rise sharply as the sensor is immersed in water again. The corresponding times of the rear sensor are $t_{1 \mathrm{r}}$ and $t_{2 \mathrm{r}}$, respectively.

Assume $N$ bubbles hit both the front and rear sensors during a sampling period $T$. For the $i^{\text {th }}$ bubble, two time intervals could be defined as follows:

$$
\begin{gathered}
\delta t_{1, i}=t_{1 f, i}-t_{1 r, i} \\
\delta t_{2, i}=t_{2 f, i}-t_{2 r, i}
\end{gathered}
$$

The mean local oil bubble velocity $u_{\mathrm{ol}}$ at the measurement position is then given by:

$$
u_{o l}=\frac{2 s}{N} \sum_{i=1}^{N} \frac{1}{\delta t_{1, i}+\delta t_{2, i}}
$$

The mean local oil volume fraction $\alpha_{\mathrm{ol}}$ can be estimated from the conductance signal from either the front or the rear sensor (Serizawa et al., 1975, p. 221). For the front sensor, $\alpha_{o l}$ is given by:

$$
\alpha_{o l}=\frac{1}{T} \sum_{i=1}^{N}\left(t_{2 f, i}-t_{1 f, i}\right)
$$

The oil volume flowrate calculation is similar with that of EIT, except here the summation is over concentric annuli and in the EIT situation it's over square pixels.

\subsection{Drift flux model}

The basic form of drift flux model in oil-water flow is: 


$$
V_{o}=C_{o} V_{m}+V_{d}^{\prime}
$$

Where $V_{\mathrm{o}}$ is the velocity of oil phase, $C_{\mathrm{o}}$ is the distribution parameter, $V_{\mathrm{m}}$ is the mixture velocity and $V_{\mathrm{d}}^{\prime}$ is the oil drift velocity.

Based on Hasan and Kabir's (1999) work, the oil drift velocity is given by:

$$
V_{d}^{\prime}=1.53 v_{c}^{\prime}\left(1-\alpha_{o}\right)^{2}
$$

Where $v_{\mathrm{c}}^{\prime}$ is the characteristic velocity, it's deduced by Harmathy (1960) in gas-liquid flows. The corresponding form in oil-water flow could be expressed as:

$$
v_{c}^{\prime}=\left[\frac{\sigma_{o w} g\left(\rho_{w}-\rho_{o}\right)}{\rho_{w}^{2}}\right]^{1 / 4}
$$

Where $\sigma_{\text {ow }}$ is oil-water surface tension, $g$ is gravitational acceleration, $\rho_{w}$ and $\rho_{\mathrm{o}}$ are densities of water and oil respectively.

In this paper, the volume fraction and velocity distributions were already obtained. The purpose of adopting drift-flux model is to calculate the relative velocity between oil and water, which is given by:

$$
v_{r}=\frac{V_{d}^{\prime}}{1-\alpha_{o}}
$$

Then further calculation of water volume flowrate could be conducted.

\section{RESULTS AND DISCUSSIONS}

According to Beck and Plaskowski (1987), the minimum acquisition time $\delta$, can be taken as twice the product of the minimum transit time of the fluid $\tau$, and the fractional velocity discrimination $\kappa$, is given in the following

$$
\kappa=\frac{\delta}{2 \tau}
$$

The discrimination resolution of the EIT system calculated from Eq.(14) in this study is about $1 \%$, which is a big advance compared with previous work (Wu et al., 2005, p. 37). The
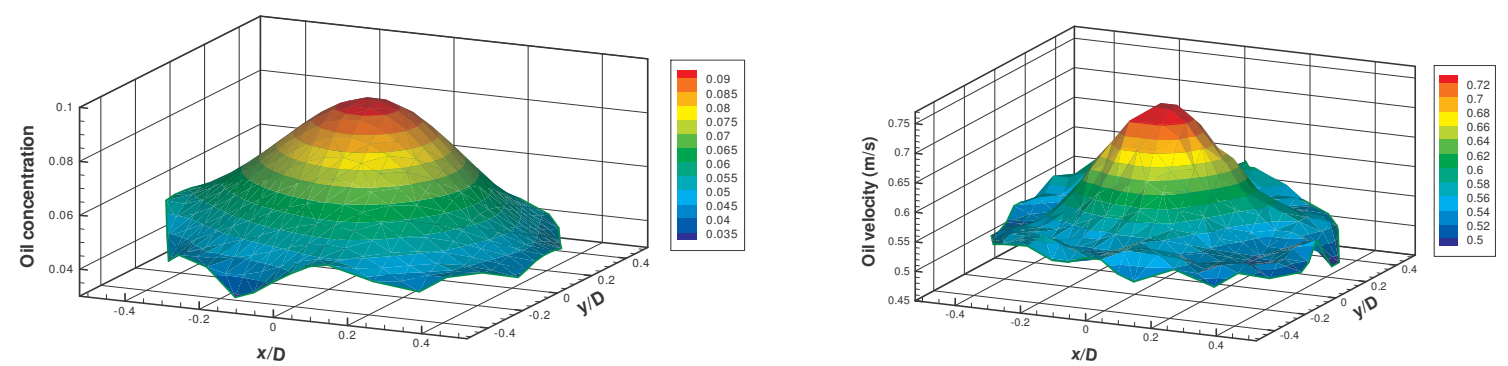

Figure 4. Oil concentration and velocity distributions from EIT $\left(Q_{\mathrm{w}}=7.5 \mathrm{~m}^{3} / \mathrm{h}, Q_{0}=0.75 \mathrm{~m}^{3} / \mathrm{h}\right)$ 

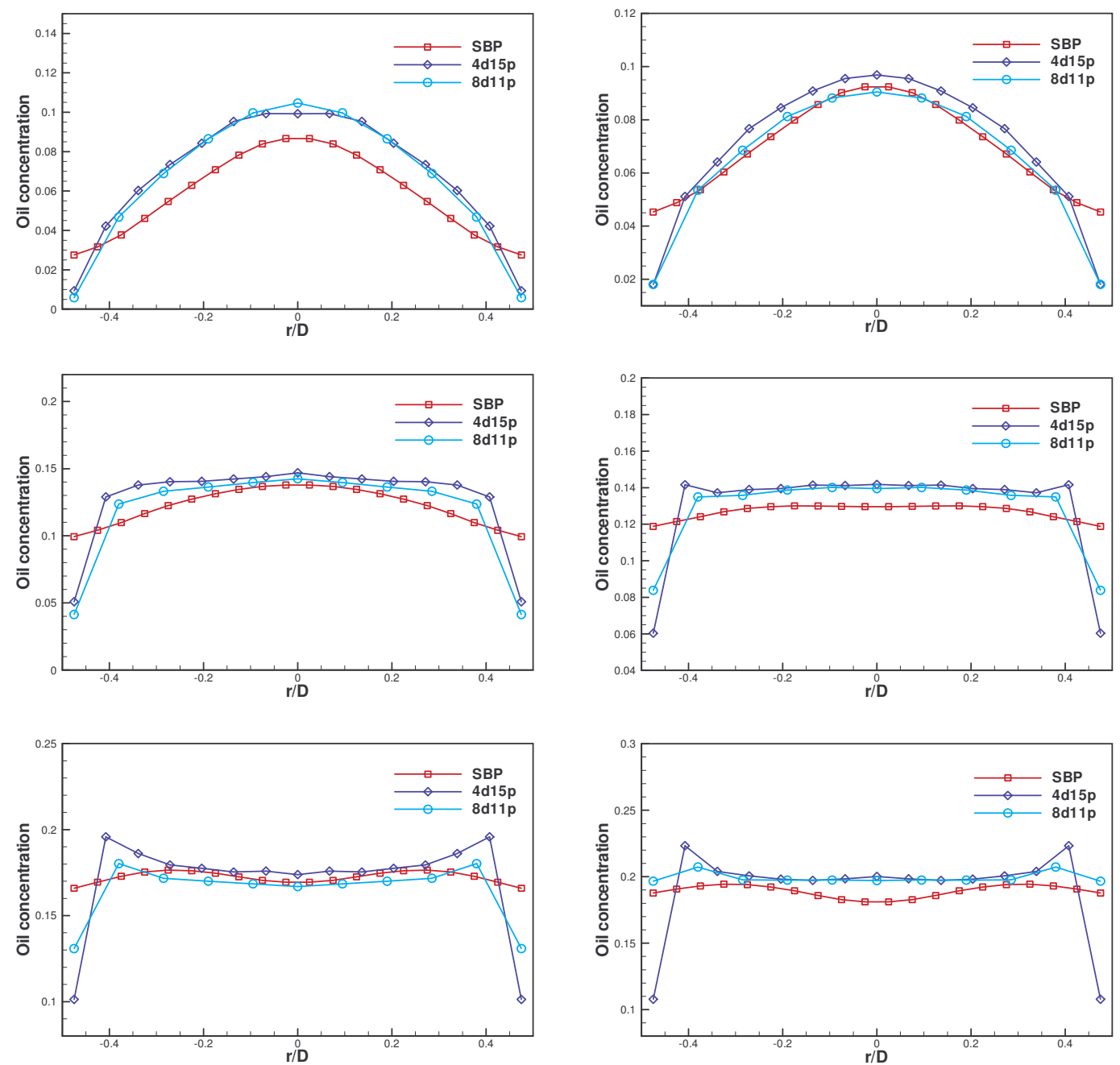

(a) $Q_{\mathrm{w}}=5 \mathrm{~m}^{3} / \mathrm{h}, Q_{\mathrm{o}}=0.5 / 1 / 1.5 \mathrm{~m}^{3} / \mathrm{h}$

(b) $Q_{\mathrm{w}}=7.5 \mathrm{~m}^{3} / \mathrm{h}, Q_{\mathrm{o}}=0.75 / 1.5 / 2.25 \mathrm{~m}^{3} / \mathrm{h}$

Figure 5. Oil concentration profiles from EIT and probe

potential of the EIT system to obtain local flow distributions can be shown in Fig. 4.

\subsection{Comparison of oil concentration and velocity profiles}

To compare with the results of probe, the cross-sectional distributions of EIT were averaged along the radial axis, and shown together with probe results in Fig.5 and Fig.6. SBP denotes the results of EIT adopting SBP reconstruction algorithm, 4d15p denotes results of probe averaged from 4 equiangled diameters with 15 equispaced measurement points on each diameter, and $8 \mathrm{~d} 11 \mathrm{p}$ denotes results of probe averaged from 8 equiangled diameters with 11 equispaced measurement points on each diameter.

Good agreements were found in both oil concentration and velocity profiles. Due to the intrusiveness of probe, the oil bubbles will be slowed down, which causes a decrease in velocity and an increase in concentration. The wall peak phenomenon is evident in the profiles from both techniques. But the intrusiveness essence of probe also comes with the simplicity of operational principle, which gives a more precise profile form. 

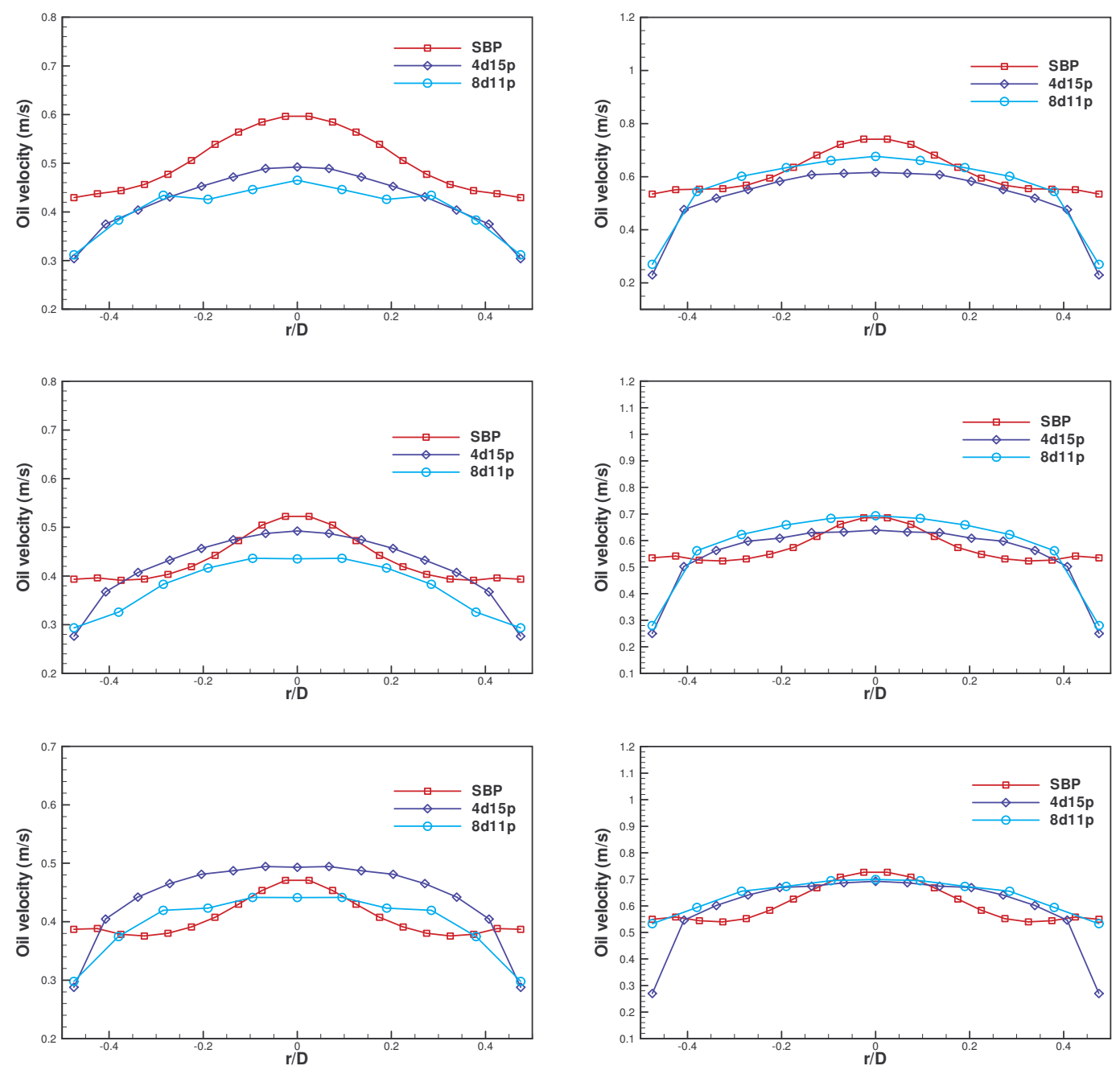

(a) $Q_{\mathrm{w}}=5 \mathrm{~m}^{3} / \mathrm{h}, Q_{\mathrm{o}}=0.5 / 1 / 1.5 \mathrm{~m}^{3} / \mathrm{h}$

(b) $Q_{\mathrm{w}}=7.5 \mathrm{~m}^{3} / \mathrm{h}, Q_{\mathrm{o}}=0.75 / 1.5 / 2.25 \mathrm{~m}^{3} / \mathrm{h}$

Figure 6. Oil velocity profiles from EIT and probe

The underestimation of concentration from EIT could also be caused by the temperature difference between the EIT sensor position and the temperature measurement position, which is located in the water storage tank where the temperature is a little lower.

The SBP algorithm results in flat concentration profiles, but it's not the case in the velocity profiles. There's a sudden drop near the center and a flat distribution near the wall, the latter could be cause by the system noise, but the former could be related with both hardware and reconstruction algorithm.

Finally, the difference is also brought by the unsteady flow status. The EIT and probe were operated in different time based on same water and oil flowrates reading from turbine flow meters. For one flow condition, EIT only needs about 9 seconds but probe needs 30 minutes, so EIT results are more easily affected. The measurement location of probe is a litter higher than EIT, which are around 25 and 20 times the inner diameter of pipe. In this range, the flow is also developing, and there's a slight difference. 

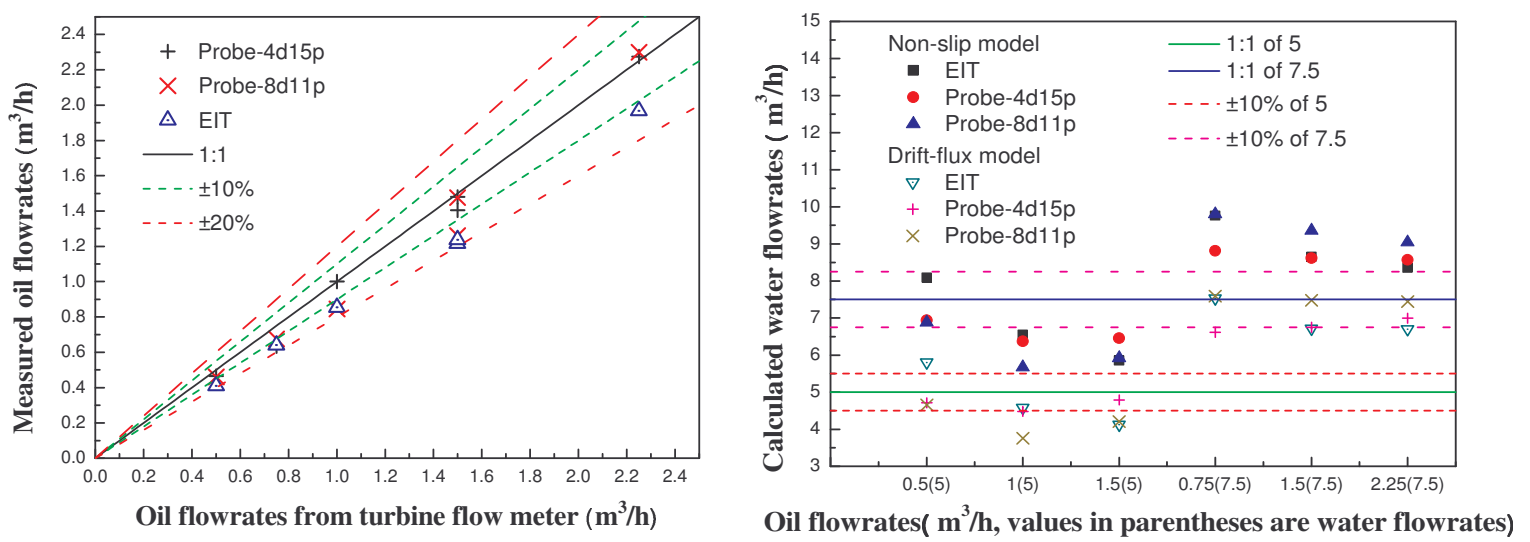

Figure 7. Comparison of oil and water volume flowrates

\subsection{Oil and water flowrates}

Oil flowrates can be calculated based on the concentration and velocity profiles. And furthermore, with the drift-flux model, the water velocity distributions then flowrates can also be calculated. The results are shown in Fig.7, in which left figure is the oil flowrates, and right figure is the water flowrates.

For the oil flowrates, better results are obtained from the probe, which are mostly situated in the error range of $10 \%$. The EIT results have an obvious underestimation, and all results are between the error range of $-10 \%$ and $-20 \%$. As stated above, it's caused by the underestimation of the concentration and the imprecise form of velocity profile.

For the water flowrates, the comparison of results from non-slip velocity model and drift-flux model are shown in the right figure in Fig.7. The former has a significant overestimation, and with the drift-flux models, most results are close to the error range of $10 \%$, which shows a significant slippage exists in the flow. Since the flow is still developing, the drift-flux model has a slight overestimation of the relative velocity, which sequentially causes lower water flowrates.

\section{Conclusions}

A study of vertical oil-in-water flow has been conducted to measure the concentration and velocity profiles, then flowrates of both phases. The following conclusions from this study are listed below:

- Good agreement was found in the oil volume fraction and velocity profiles between EIT and probe.

- Due to the difference in operational principle and unsteady flow conditions, probe results have a better description of the profile form.

- The accuracy of concentration calculation with EIT remarkably relies on the conductivity of water, which caused an underestimation of oil concentration. 
- Significant slippage was found in the oil-in-water flow, and drift-flux model is an effective approach to describe it.

- A reconstruction algorithm for EIT with improved precision and proper speed is needed to obtain more accurate results in on-line measurement.

- EIT is a non-intrusive technique with the capability to obtain detailed flow information. It's a promising approach for two-phase flow measurement with appropriate modelling technique and calibration.

\section{ACKNOWLEDGEMENTS}

The authors would like to acknowledge Marie Curie Actions (EU FP6) for supporting this work.

\section{REFERENCES}

Beck, M.S. and A. Plaskowski, 1987, Cross Correlation Flowmeters: Their Design and Application, Adam Hilger, Bristol.

Dickin, F.J., R.A. Williams and M.S. Beck, 1993, Determination of Composition and Motion of Multicomponent Mixtures in Process Vessels using Electrical Impedance Tomography -I. Principles and Process Engineering Applications, Chem. Eng. Sci. 48, 1883.

Flores, J.G., C. Sarica, T.X. Chen and J.P. Brill, 1998, Investigation of Holdup and Pressure Drop Behavior for Oil-Water Flow in Vertical and Deviated Wells, JERT, Trans. ASME $120,8$.

Harmathy, T.Z., 1960, Velocity of Large Drops and Bubbles in Media of Infinite and Restricted Extent, AICHE J. 6, 281.

Hasan, A.R. and C.S. Kabir, 1999, A Simplified Model for Oil/Water Flow in Vertical and Deviated Wellbores, SPE Prod. \& Fac. 14, 56.

Lucas, G.P., R. Mishra and N. Panayotopoulos, 2004, Power Law Approximations to Gas Volume Fraction and Velocity Profiles in Low Void Fraction Vertical Gas-liquid Flows, Flow Meas. Instrum. 15, 271.

Maxwell, J.C., 1881, A Treatise on Electricity and Magnetism, Clarendon Press, Oxford.

Serizawa, A., I. Kataoko and I. Michiyoshi, 1975, Turbulence Structure of Air-Water Bubbly Flow-I. Measuring Techniques, Int. J. Multiphase Flow 2, 221.

Wang, M., R. Mann and F.J. Dickin, 1999, Electrical Resistance Tomographic Sensing Systems for Industrial Applications, Chem. Eng. Comm. 175, 49.

Wang, M., T.F. Jones and R.A. Williams, 2003, Visualization of Asymmetric Solids Distribution in Horizontal Swirling Flows using Electrical Resistance Tomography, Trans. IChemE. 81, 854.

Wang, M., Y. Ma, N. Holliday, Y. Dai, R.A. Williams and G.P. Lucas, 2005, A Highperformance EIT System, IEEE Sens. J. 5, 289.

Wu, Y., H. Li, M. Wang and R.A. Williams, 2005, Characterization of Air-water Two-phase Vertical Flow by using Electrical Resistance Imaging, Can. J. Chem. Eng. 83, 37.

Zuber, N., and J.A. Findlay, 1965, Average volumetric Concentration in Two-Phase Flow Systems, J. Heat Tran., Trans. ASME 87, 453. 